\title{
Response Suppression of Multiple Hinged Floating Structures by Using Rubber Cushion
}

\author{
Ye Lu, ${ }^{1}$ Qijia Shi ${ }^{D},{ }^{2}$ Yuchao Chen, ${ }^{1}$ Wenhui Zheng, ${ }^{1}$ and Ye Zhou ${ }^{1}$ \\ ${ }^{1}$ China Ship Scientific Research Center, Wuxi 214082, China \\ ${ }^{2}$ Schools of Hydraulic Engineering, Changsha University of Science \& Technology, Changsha 410114, China \\ Correspondence should be addressed to Qijia Shi; scottsqj@hnu.edu.cn
}

Received 8 April 2021; Revised 21 July 2021; Accepted 14 August 2021; Published 27 August 2021

Academic Editor: Vasudevan Rajamohan

Copyright (c) 2021 Ye Lu et al. This is an open access article distributed under the Creative Commons Attribution License, which permits unrestricted use, distribution, and reproduction in any medium, provided the original work is properly cited.

\begin{abstract}
A brand-new rubber cushion is proposed in this paper, which is installed between hinged floating modules in order to reduce the relative motion of the pitch; meanwhile, the cushion can be used as a fender for anti-impact in the docking process. Using the linear wave potential method, the structural dynamic model is formulated where the equivalent stiffness matrix for the rubber cushion is obtained by an integrating method employing linear assumption in addition to considering the heterogeneity of rubber. A numerical analysis is presented for a two-module semisubmersible floating structure. The hydrodynamic responses and connector loads of the floating structures with a rubber cushion are analyzed by using the frequency domain approach in both regular and irregular waves. The topological design and stiffness parameter selection of the rubber cushion is studied. This work may provide a new idea for suppressing the pitch motion of multiple hinged floating structures.
\end{abstract}

\section{Introduction}

Owing to the growth in population and the expansion of urban cities in coastal areas, it needs land reclamation from the sea for human activities and freeing valuable land sites for commercial and housing projects. With these points in mind, very large floating structures (VLFSs) have been proposed to acquire ocean space, which can be used in different practical applications such as floating bridges, emergency bases, airports, cities, energy farms, fuel storage facilities [1-6], etc. The design of a VLFS with efficient safety and performance is the key issue for successful implementations, operations, and further development [7].

Since the VLFSs perform in a harsh ocean environment that changes with time, it is very important to perform dynamic analysis of the VLFS to improve the design for meeting a required safety and span of a lifetime. In the mattype VLFS, draft to length ration is small enough, and the hydroelastic theory is widely used to predict dynamic behaviors. In this class of problem, a common approach is modeling the entire VLFS as a single plate, and the study is investigated on the basis of the classical thin or thick plate theory [8]. The VLFSs linked with connectors are the best choice owing to the convenience in construction, transportation, and deployment. Thus, the connector becomes a key element for the design of the VLFS [9]. Hinge connection (namely, semirigid connector), which allows the relative pitch motion of the modules, is used to reduce the connector loads and the bending moment on cross section of the modules. For a floating barge structure serially and longitudinally connected by a hinge connector, Newman [10] analyzed the relative motions between adjacent modules and the shear loads in the connectors by using the generalized modes. The results showed that, in general, the largest loads occur on the interior hinges and the maximum hinge loads increase with the increase of the number of modules. Yoon et al. [11] proposed a numerical method to analyze modular floating structures coupled with longitude and/or transverse directional multiple hinge connectors. The deflection of the plate structures and maximum bending moment are investigated via the directly coupled governing equations discretized by the finite element method for plates and the boundary element method for fluid. The numerical results showed that the number of hinge connections has a 
great effect on the deflection of the floating plate structures. Instead of the box-shaped pontoon as reviewed above, the semisubmersible type is an optimal choice in order to reduce the hydrodynamics responses in the deep-sea area. Lee and Newman [12] studied the effects of linear or second-order waves on VLFS consisting of five modules connected by simple hinge connectors. The vertical motions, structural defections, and hinge shear forces were analyzed in different wave conditions where the structural stiffness parameters were considered to evaluate the effect of the hydroelasticity.

It is obvious that the study on the articulated floating structures reviewed above adopted the pure hinge connection which can ensure the continuity of displacements at the connector points of adjacent decks. But there is no restraint of relative pitch motions of adjacent modules. However, stringent tolerance on the deformation of the VLFS is significant in some applications, such as floating airports. Therefore, the reduction of the hydroelastic response of modular floating structures is significantly concerned. Except for liquid-contained rectangular tanks [13], several approaches have been proposed for mitigating the hydroelastic response of the VLFS under wave action, such as arranging breakwater in the periphery of VLFS [14], imposing control strategy [15], and equipping with wave energy conversion system. Another innovative method for the reduction of the hydroelastic response of the VLFS is to use flexible-hinge hybrid connectors instead of pure hinge connectors [16]. Fu et al. [17] presented a hydroelastic theory of floating structures consisting of two flexible, interconnected modules with rotation stiffness. In their paper, the effects of connector and module stiffness as well as the behaviors of the bending moment distribution on the hydroelastic response of the structure were studied. The numerical analysis showed that the rotation stiffness of the connector has a significant impact on the hydroelastic response of the structure. With the purpose of minimum hydroelastic response, Riyansyah et al. [18] studied the parameters' design of the connectors for a two-module floating beam structure based on the frequency domain approach in which the BEM and the FEM are applied to solve the boundary value problem for fluid motion and the dynamic problem for beam motion, respectively. They found the optimum location and rotational stiffness of the connector between the two-module beam structures. Zhao et al. [19] studied the influence law of the connection conditions on the hydroelastic response of the floating structures coupled with torsion spring connectors. Their numerical results showed that the hydroelastic response of the plate can be mitigated effectively by choosing the appropriate spring stiffness and hinge position. Reviewing the earlier research works as mentioned above and many others not listed here, we can conclude that almost all of the modeling methods adopt the idealized torsion stiffness instead of concrete physical structure for hinged connection. In addition, the docking process of multiple modules floating system is complicated due to the changeable and rough sea conditions. How to avoid collision damage of the floating structures in the docking process is one of the most important concerns.
In this study, we propose installing rubber cushions between two consecutive hinged floating structures to reduce the relative pitch motion and ensure the smoothness between adjacent modules; they can also be used as an antiimpact buffer pad in the docking process. Based on linear wave theory, the dynamic model is formulated in which the equivalent stiffness matrix for the rubber cushions is derived by integrating method based on linear assumption considering the heterogeneity of rubber. A numerical experiment of a two-module semisubmersible structure is carried out. The performance of the rubber cushion on the connector loads and the hydrodynamic responses are analyzed by means of the frequency domain approach in regular and irregular waves. The topological design and stiffness parameter selection of the rubber cushion is evaluated. At last, the strength of the rubber cushion is computed. The results obtained in this paper can provide a theoretical base for designing the hinged floating platform systems.

\section{Model of Multiple Floating Modules Coupled with Hinges and Rubber Cushion}

For the modeling of the present problem, two coordination systems are set up firstly, shown in Figure 1. In the global coordinate system $(X, Y, Z)$, the $X-Y$ plane is on the calm water surface, the $X$-axis is consistent with the longitudinal direction of the module, and the $Z$-axis is vertical water surface upward. The local coordination system $\left(x_{i}, y_{i}, z_{i}\right)$ is fixed on the $i$-th module, where the origin point sets on the center of gravity (CG) of the module. The $x_{i}, y_{i}, z_{i}$ axes coincide with the directions of $X, Y, Z$ axes, respectively.

The rigid module and flexible connector (RMFC) model in which each module has only six degrees of freedom (DOFs) is used for simulation. The displacements and rotations at the CG of each module are the usual choice for the DOFs, shown in Figure 1. In this paper, the small displacement assumption is adopted for initial design and parameter studies such that linear kinematic theory is valid.

2.1. Modeling of Single Floating Module. The governing equations of the motion for the floating module $i$ are formulated based on Newton's second law:

$$
\mathbf{M}_{i} \ddot{\mathbf{X}}_{i}+\mathbf{S}_{i} \mathbf{X}_{i}=\mathbf{F}_{i, W}+\mathbf{F}_{i, C}+\mathbf{F}_{i, R},
$$

where $\mathbf{X}_{i}=\left[x_{i}, y_{i}, z_{i}, \alpha_{i}, \beta_{i}, \gamma_{i}\right]^{T}$ denotes the displacement vector of module $i$. The symbols $x_{i}, y_{i}, z_{i}, \alpha_{i}, \beta_{i}$, and $\gamma_{i}$ correspond with surge, sway, heave, roll, pitch, and yaw (see Figure 1), and the superscript symbol $T$ denotes the transpose. The mass matrix $\mathbf{M}_{i}$ is defined as

$$
\mathbf{M}_{i}=\left[\begin{array}{cccccc}
m_{i} & 0 & 0 & 0 & 0 & 0 \\
0 & m_{i} & 0 & 0 & 0 & 0 \\
0 & 0 & m_{i} & 0 & 0 & 0 \\
0 & 0 & 0 & J_{i x} & 0 & 0 \\
0 & 0 & 0 & 0 & J_{i y} & 0 \\
0 & 0 & 0 & 0 & 0 & J_{i z}
\end{array}\right],
$$




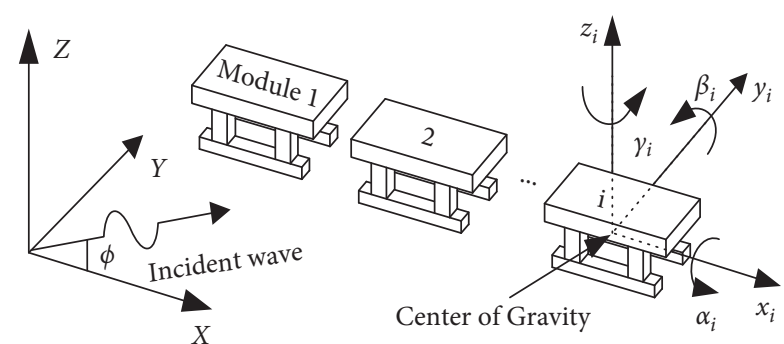

Figure 1: Sketch of the coordination systems, in which $(X, Y, Z)$ denotes the global coordinate, $\left(x_{i}, y_{i}, z_{i}\right)$ illustrates the local coordinate, and $\phi$ is the incident wave angle.

where $m_{i}$ denotes the mass of the $i$-th floating module. The quantities $J_{i x}, J_{i y}$, and $J_{i z}$ represent the moments of inertia of the $i$-th module about the $x_{i}, y_{i}$, and $z_{i}$ axes, respectively, where

$$
\begin{aligned}
J_{i x} & =\iint_{V} x_{i}^{2} \mathrm{~d} m+\iint_{V} z_{i}^{2} \mathrm{~d} m \\
J_{i j} & =\iint_{V} z_{i}^{2} \mathrm{~d} m+\iint_{V} x_{i}^{2} \mathrm{~d} m, J_{i z}=\iint_{V} x_{i}^{2} \mathrm{~d} m+\iint_{V} y_{i}^{2} \mathrm{~d} m .
\end{aligned}
$$

In equation (1), $\mathbf{S}_{i}$ is the hydrostatic restoring coefficient matrix, which is given as follows [20]:

$$
\mathbf{S}_{i}=-\left[\begin{array}{cccccc}
0 & 0 & 0 & 0 & 0 & 0 \\
0 & 0 & 0 & 0 & 0 & 0 \\
0 & 0 & S_{i z} & 0 & 0 & 0 \\
0 & 0 & 0 & M_{i \alpha} & 0 & 0 \\
0 & 0 & 0 & 0 & M_{i \beta} & 0 \\
0 & 0 & 0 & 0 & 0 & 0
\end{array}\right],
$$

where the coefficients $S_{i z}, M_{i \alpha}$, and $M_{i \beta}$ are given by

$$
\begin{aligned}
& S_{i z}=-\rho g A_{i w}, \\
& M_{i \alpha}=-\rho g V_{i} z_{i b}-\rho g \iint_{A_{i w}} y_{i}^{2} \mathrm{~d} A, \\
& M_{i \beta}=-\rho g V_{i} z_{i b}-\rho g \iint_{A_{i w}} x_{i}^{2} \mathrm{~d} A,
\end{aligned}
$$

In equations (5)-(7), $g$ denotes the acceleration of gravity, $z_{i b}$ indicates the $z$-coordinates of the center of buoyancy (CB) of module $i, A_{i w}$ is the area in contact with water, and $V_{i}$ represents the displacement.

The three quantities $\mathbf{F}_{i, W}, \mathbf{F}_{i, C}$, and $\mathbf{F}_{i, R}$ in equation (1) denote the wave load, connector load, and the load produced by the rubber cushion, respectively, to be derived later.

2.2. Hydrodynamic Model. By applying linear wave theory, the wave potential is expressed as $\Phi=\operatorname{Re}\left[\varphi e^{- \text {\&ImaginaryI } ; \omega t}\right]$ where $\omega$ indicates regular frequency, and $\varphi$ denotes the spatial velocity potential. In the system with $N$ numbers of floating modules, the space velocity potential of the whole system can be written as

$$
\varphi=\varphi_{I}+\varphi_{D}+i \omega \sum_{i=1}^{N} \sum_{q=1}^{6} \bar{X}_{i}^{q} \varphi_{i}^{q}
$$

in which $i=\sqrt{-1}$, and $\varphi_{I}$ and $\varphi_{D}$ denote incident and diffraction potentials, respectively. Furthermore, $\varphi_{i}^{q}$ denotes the unit radiation potential due to the $q$-th modal motion of the $i$-th module. $\bar{X}_{i}^{q}$ represents the complex amplitude of the $i$-th module which satisfied the displacement vector $\mathbf{X}_{i}=\bar{X}_{i} e^{-\& I m a g i n a r y I ; \omega t}$, and $\bar{X}_{i}=\left[\bar{X}_{i}^{1}, \bar{X}_{i}^{2}, \ldots, \bar{X}_{i}^{6}\right]$.

For the present problem, the foregone incident potential $\varphi_{I}$ can be written as

$$
\varphi_{I}=\frac{i g a}{\omega} \frac{\cosh k(z+h)}{\cosh k h} \exp [i k\{x \cos \phi+y \sin \phi\}],
$$

where $a$ denotes the wave amplitude, $h$ is the water depth, $\phi$ indicates the incident wave angle, and $k$ is the wavenumber which satisfied the dispersion relationship $\omega^{2}=\operatorname{gktanh}(k h)$.

The diffraction and unit radiation potential $\varphi_{D}$ and $\varphi_{i}^{q}$ satisfy the Laplace equation and the linearized boundary conditions, which are given by

$$
\left\{\begin{array}{l}
\nabla^{2} \varphi_{D}=0 \\
\frac{\partial \varphi_{D}}{\partial z}-\frac{\omega^{2}}{g}=0 \\
\frac{\partial \varphi_{D}}{\partial n}=-\frac{\partial \varphi_{I}}{\partial n} \\
\frac{\partial \varphi_{D}}{\partial z}=0, \quad z=-h, \\
\frac{\lim _{\longrightarrow}}{r} \sqrt{r}\left(\frac{\partial \varphi_{D}}{\partial r}-\frac{i \omega^{2}}{g \varphi_{D}}\right)=0 \\
\frac{\partial \varphi_{i}^{q}}{\partial n}, \quad(i \neq m), \\
\frac{\partial \varphi_{i}^{q}}{\partial \varphi_{i}^{q}}=0, \\
\frac{\partial \varphi_{i}^{q}}{\partial z}-\frac{\omega^{2}}{g \varphi_{i}^{q}}=0, \\
S_{i}=0, \quad n_{i}^{q}, \\
\left.\frac{\partial \varphi_{i}^{q}}{\partial r}-\frac{i \omega^{2}}{g \varphi_{i}^{q}}\right)=0
\end{array}\right.
$$


where $n_{i}^{q}$ is the generalized outward normal vector of the $q$-th modal of the $i$-th module. $r$ denotes the displacement between the field point and source point.
The wave force of the $i$-th module in the $p$-th modal direction of the $i$-th modules can be obtained by using Bernoulli's equation and is given by

$$
\begin{aligned}
\bar{W}_{i}^{p} & =\iint_{S} \frac{\partial \phi_{i}^{p}}{\partial n}\left[-\mathrm{i} \omega \rho\left(\phi_{I}+\phi_{D}+\mathrm{i} \omega \sum_{j=1}^{N} \sum_{q=1}^{6} \bar{X}_{j}^{q} \phi_{j}^{q}\right)\right] \mathrm{d} s, \\
& =\iint_{S_{i}} n_{i}^{p}\left[\left[-\mathrm{i} \omega \rho\left(\phi_{I}+\phi_{D}+\mathrm{i} \omega \sum_{j=1}^{N} \sum_{q=1}^{6} \bar{X}_{j}^{q} \phi_{j}^{q}\right)\right]\right] \mathrm{d} s .
\end{aligned}
$$

The total wave force as in equation (11) can be expressed as a vector form as follows:

$$
\mathbf{F}_{i, W}=\overline{\mathbf{F}}_{i, W} e^{-\mathrm{i} \omega t}-\sum_{j=1}^{N}\left(\mathbf{A}_{i j} \ddot{\mathbf{X}}_{j}+\mathbf{B}_{i j} \dot{\mathbf{X}}_{j}\right)
$$

where the excitation force term $\bar{F}_{i, W} e^{-i \omega t}$ in equation (12) is derived by scattering wave potential $\varphi_{S}=\varphi_{I}+\varphi_{D}$ and the other term denotes the radiation resistance of wave. $\mathbf{A}_{i j}$ and $\mathbf{B}_{i j}$ denote added mass and radiation damping matrices, respectively.

Note that the software THAFTS (Three-dimensional Hydroelastic Analysis of Floating and Translating Structure) is used to solve the boundary value problem (10) for the exciting forces and the hydrodynamic coefficients in this paper.

\subsection{Modeling of Hinge Connector with Rubber Cushion.} In this study, two hinge connectors with rubber cushions are arranged between two adjacent modules, as shown in Figure 2.

The rubber cushion is shown in Figure 2(b) which consists of a mounting base, multilayer rubber materials, and two clamps. The mounting base of the rubber cushion is mounted on the left module and the clamps of the rubber cushion dock into the locking device of another module. The beveled clamps of the cushions and beveled chucks of the locking device are convenient for quick installation and release for special circumstances.

Considering the linear assumption of the small displacements described above, the principle of linear superposition is applicative for the modeling of the hinge connector and rubber cushion. In the modeling process, only one part (either hinge or rubber cushion) of the connection is effective while the other is removable. Then, the whole model of the connection is obtained using the linear superposition method. Though it is worth noticing that the rubber material has nonlinear and damping properties firstly, we want to obtain the dynamic behaviors of the whole system using the simple linear model. Then, we carry out the strength analysis by using nonlinear FEM.

Firstly, the model of the hinge connector is derived. In our model, we use two spherical hinges to represent the two hinge connectors. One spherical hinge connector can restrain three translational displacements, and thus, six translational displacements will be constrained by two spherical hinges. However, there are only five constraint equations among adjacent modules. Therefore, applying two spherical hinges in the connection among adjacent modules results in an overconstrained problem. Thus, we have to release a translational displacement to solve this problem. Hence, the right hinge is allowed to displace along $x, y$, and $z$ directions, whereas the left hinge is free to move along $x$ and $z$ directions. Indeed, the equally shared transversal loads in both hinges (due to the symmetry) will be artificially transferred to the right hinge. Considering the symmetry and rigidity of the whole floating system, this transformation for the transversal load does not affect the yaw moment at the CG of a module. The symmetry still remains in responses. The connection model is shown in Figure 3.

The force vectors $\mathbf{F}_{c i}^{L}$ and $\mathbf{F}_{c i}^{R}$ act on the pseudospherical hinge and spherical hinge, respectively, which are placed between module $i$ and module $(i+1)$. Thus, the force vector imposed on the CG of module $i$ induced by the spherical hinge connector can be expressed as

$$
\begin{aligned}
& \mathbf{F}_{i}^{R}=\mathbf{D}_{S i}^{R T} \mathbf{F}_{c i}^{R}-\mathbf{D}_{H i}^{R T} \mathbf{F}_{c(i-1)}^{R}, \quad 1<i<N, \\
& \mathbf{F}_{i}^{R}=\mathbf{D}_{S i}^{R T} \mathbf{F}_{c i}^{R}, \quad i=1, \\
& \mathbf{F}_{i}^{R}=-\mathbf{D}_{H i}^{R T} \mathbf{F}_{c(i-1)}^{R}, \quad i=N .
\end{aligned}
$$

In addition, the force vector of the pseudospherical hinge connector imposing on the CG of module $i$ is similarly expressed as

$$
\begin{aligned}
& \mathbf{F}_{i}^{L}=\mathbf{D}_{S i}^{L T} \mathbf{F}_{c i}^{L}-\mathbf{D}_{H i}^{L T} \mathbf{F}_{c(i-1)}^{L}, \quad 1<i<N, \\
& \mathbf{F}_{i}^{L}=\mathbf{D}_{S i}^{L T} \mathbf{F}_{c i}^{L}, \quad i=1, \\
& \mathbf{F}_{i}^{L}=-\mathbf{D}_{H i}^{L T} \mathbf{F}_{c(i-1)}^{L}, \quad i=N .
\end{aligned}
$$

The symbols $\mathbf{D}_{S i}^{R}$ and $\mathbf{D}_{H i}^{R}$ as in equation (13) and $\mathbf{D}_{S i}^{L}$ and $\mathbf{D}_{H i}^{L}$ as in equation (14) denote the constraint matrices of module $i$ for spherical hinge connector and pseudospherical hinge connector, respectively, and the subscript $S$ or $H$ denotes the connection point on the stem or head of the module. The general form of the constraint matrix for spherical hinge connector can be expressed as 




(a)

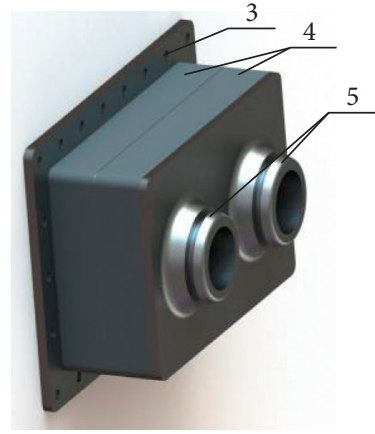

(b)

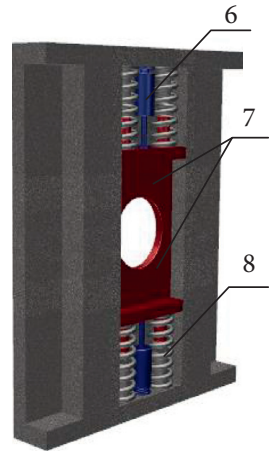

(c)

Figure 2: Conceptual model of floating modules connected with hinges and rubber cushions: (a) floating modular structures, (b) rubber cushion, and (c) locking device of a floating module (1, hinge connector; 2 , rubber cushion; 3 , mounting base; 4 , rubber; 5 , clamp; 6 , hydraulic cylinder; 7 , chuck; 8 , spring).

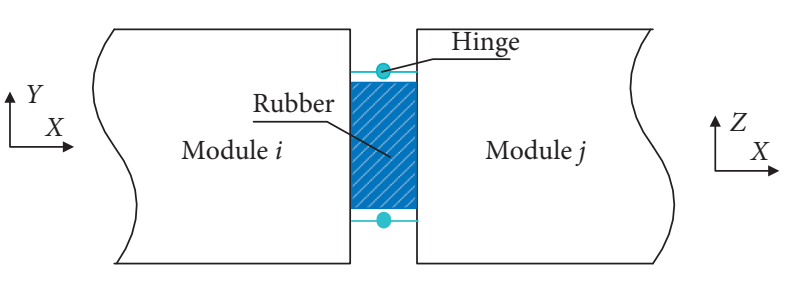

(a)

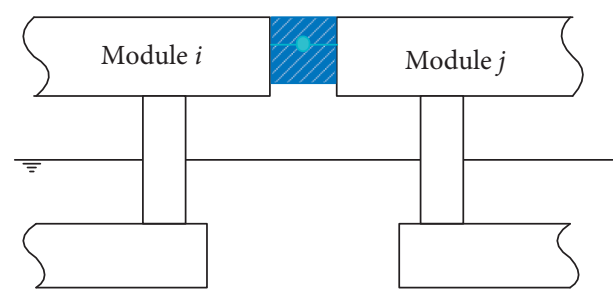

(b)

FIgURE 3: Sketch of floating modules coupled with hinges and rubber cushion.

$$
\mathbf{D}_{\Theta i}^{R}=\left[\begin{array}{cccccc}
1 & 0 & 0 & 0 & z_{\Theta i}^{R} & -y_{\Theta i}^{R} \\
0 & 1 & 0 & -z_{\Theta i}^{R} & 0 & x_{\Theta i}^{R} \\
0 & 0 & 1 & y_{\Theta i}^{R} & -x_{\Theta i}^{R} & 0
\end{array}\right] .
$$

For the pseudospherical hinge connection, the constraint matrix is

$$
\mathbf{D}_{\Theta i}^{L}=\left[\begin{array}{cccccc}
1 & 0 & 0 & 0 & z_{\Theta i}^{L} & -y_{\Theta i}^{L} \\
0 & 0 & 1 & y_{\Theta i}^{L} & 0 & x_{\Theta i}^{L}
\end{array}\right],
$$

where the subscript $\Theta$ indicates $S$ or $H$ and $\left(x_{\Theta i}^{R}, y_{\Theta i}^{R}, z_{\Theta i}^{R}\right)$ and $\left(x_{\Theta i}^{L}, y_{\Theta i}^{L}, z_{\Theta i}^{L}\right)$ indicate the coordinates in the local coordinate system of the connection point of the $i$ th module for the spherical hinge and pseudospherical hinge, respectively.

The total force applied to the $i$-th module by the hinged connector can be written as

$$
\mathbf{F}_{i, C}=\mathbf{F}_{i}^{L}+\mathbf{F}_{i}^{R}
$$

The rigid hinge connector should satisfy the displacement continuity condition. For the spherical hinge connection, the equation can be written as

$$
D_{S i}^{R} \mathbf{X}_{i}-D_{H(i+1)}^{R} \mathbf{X}_{i+1}=0, \quad i=1,2, \ldots, N-1 .
$$

And for the pseudospherical hinge connector, it yields

$$
D_{S i}^{L} \mathbf{X}_{i}-D_{H(i+1)}^{L} \mathbf{X}_{i+1}=0, \quad i=1,2, \ldots, N-1 .
$$

Now, the mechanical model of the twin hinge connectors and the constitutive model for the rubber cushion are both derived. Considering the principle of linear superposition, the constraint of the hinge connector between the adjacent modules is freed in the derivative process of the rubber cushion.

It is assumed that the rubber cushion is only deformed by tension and compression (the shearing deformation is ignored because the hinges take the total shear loads). It is worth noticing that the rubber is essentially a nonlinear material. The exact model should be established based on the nonlinear finite element method (FEM) or other numerical methods for structure modeling, while the entire nonlinear mechanical model in the time domain is unbearable for the global hydrodynamic analysis for the multiple floating structures. Some simplified methods are necessary for the dynamic response prediction at the early stage. Thus, another assumption is that the cushion possesses linear property in stiffness. For this assumption, the rubber cushion only provides rubber constraints for adjacent modules in the directions of the surge, pitch, and yaw. In what follows, the equivalent stiffness of the rubber cushion will be formulated based on the assumption of linearity and small rotation angle.

Based on the above hypothesis, when module $i$ only has pitch motion $\beta_{i}$, module $j$ has only the pitch motion $\beta_{j}$ (see Figure 4). Surge displacements for the points $P_{i}$ and $P_{j}$ on the modules $i, j$ whose distance from the CG is $Z$ can be written as 




FIgURE 4: The position and displacement relationship between adjacent modules for rubber element (the dashed or solid line triangles denote the initial or the deformation position and blue lines indicate rubber element).

$$
\begin{aligned}
& u_{i}=Z \beta_{i}, \\
& u_{j}=Z \beta_{j} .
\end{aligned}
$$

Considering the infinitesimal volume (or element) for the rubber cushion between the two points $P_{i}, P_{j}$ and rubber has linear tension-compression property with stiffness for unit area $k$, thus the longitudinal force of a rubber element can be expressed as

$$
\mathrm{d} F_{x i j}=k\left(u_{j}-u_{i}\right) \mathrm{d} S=k Z\left(\beta_{j}-\beta_{i}\right) \mathrm{d} S,
$$

where $\mathrm{d} S$ denotes the area of the element surface. The longitudinal force $F_{x i j}$, on the whole rubber cushion can be obtained from the following relation:

$$
F_{x i j}=\int \mathrm{d} f=\int_{Y_{r}} \int_{Z_{r}} k Z\left(\beta_{j}-\beta_{i}\right) \mathrm{d} z \mathrm{~d} y .
$$

The force exerted by the rubber cushion on the module will cause torques to the CG of modules. The torque due to the infinitesimal volume for the rubber cushion can be formulated as

$$
\mathrm{d} M_{\beta i j}=k\left(u_{j}-u_{i}\right) Z_{y} \mathrm{~d} S=k Z Z_{y i}\left(\beta_{j}-\beta_{i}\right) \mathrm{d} S,
$$

where $Z_{y i}$ denotes the force arm of the longitudinal force acting on the CG of $i$-th module and is of the form $Z_{y i}=Z \cos \beta_{i}$. It is assumed that the rotational angle $\beta_{i}$ is small enough (i.e., $\cos \beta_{i}=1$ ). Thus, equation (23) is reduced to

$$
\mathrm{d} M_{\beta i j}=k Z^{2}\left(\beta_{j}-\beta_{i}\right) \mathrm{d} S .
$$

The torque on the whole rubber cushion can be obtained from equation (24) and is as follows:

$$
M_{\beta i j}=\left(\beta_{j}-\beta_{i}\right) \int_{Y_{r}} \int_{Z_{r}} k Z^{2} \mathrm{~d} Z \mathrm{~d} Y
$$

Introducing equivalent compression-tension and twist stiffness,

$$
\begin{aligned}
k_{x \beta e} & =\int_{Y_{r}} \int_{Z_{r}} k Z \mathrm{~d} Z \mathrm{~d} Y, \\
k_{\beta e} & =\int_{Y_{r}} \int_{Z_{r}} k Z^{2} \mathrm{~d} Z \mathrm{~d} Y .
\end{aligned}
$$

Similarly, the equivalent compression-tension and twist stiffness due to yaw motion can be formulated as

$$
\begin{gathered}
k_{x \gamma e}=-\int_{Z_{r}} \int_{Y_{r}} k Y \mathrm{~d} Y \mathrm{~d} Z, \\
k_{\gamma e}=\int_{Z_{r}} \int_{Y_{r}} k Y^{2} \mathrm{~d} Y \mathrm{~d} Z,
\end{gathered}
$$

where the symbol $Y$ denotes the horizontal distance from the CG.

If the adjacent modules have only relative surge, the equivalent stiffness can be derived as

$$
\begin{aligned}
k_{x e} & =\int_{Z_{r}} \int_{Y_{r}} k \mathrm{~d} Y \mathrm{~d} Z, \\
k_{\beta x e} & =\int_{Y_{r}} \int_{Z_{r}} k Z \mathrm{~d} Y \mathrm{~d} Z, \\
k_{\gamma x e} & =-\int_{Y_{r}} \int_{Z_{r}} k Y^{2} \mathrm{~d} Y \mathrm{~d} Z,
\end{aligned}
$$

where $Y_{r}, Z_{r}$ denote the integral direction along the width and height of the rubber cushion.

Thus, the connector force can be expressed as

$$
\mathbf{F}_{i, R}=\mathbf{F}_{i(i+1)}^{R}+\mathbf{F}_{i(i-1)}^{R}=\mathbf{K}^{R}\left(\mathbf{X}_{i+1}-\mathbf{X}_{i}\right)+\mathbf{K}^{R}\left(\mathbf{X}_{i-1}-\mathbf{X}_{i}\right),
$$

where the matrix $\mathbf{K}^{R}$ indicates the equivalent rubber stiffness matrix:

$$
\mathbf{K}^{R}=\left[\begin{array}{cccccc}
k_{x e} & 0 & 0 & 0 & k_{x \beta e} & k_{x \gamma e} \\
0 & 0 & 0 & 0 & 0 & 0 \\
0 & 0 & 0 & 0 & 0 & 0 \\
0 & 0 & 0 & 0 & 0 & 0 \\
k_{\beta x e} & 0 & 0 & 0 & k_{\beta e} & 0 \\
k_{\gamma x e} & 0 & 0 & 0 & 0 & k_{\gamma e}
\end{array}\right] .
$$

It should be noticed that rubber is a kind of polymer composite material and its stiffness $k$ depends on the proportion of base materials. On the other hand, the rubber cushion always consists of rubber material and a metal skeleton. The stiffness of the rubber cushion can be adjusted by the structural design of the metal skeleton.

2.4. Dynamic Modeling of Hinged Floating Modules with Rubber Cushions. If $N$ modules are serially connected with hinge connectors and rubber cushions, the governing equation of motions can be deduced from integrating the single floating body equation of motion as in equation (1) whereas the wave force, mechanics model of hinge connector, mechanics model of the rubber cushion, and the constraint conditions of hinge connector can be obtained from equations (12), (17)-(19), and (29), respectively. Thus, the system of equations is as follows: 


$$
\begin{aligned}
\mathbf{M}_{i} \ddot{\mathbf{X}}_{i}+\sum_{j=1}^{N}\left(\mathbf{A}_{i j} \ddot{\mathbf{X}}_{j}+{ }_{\mathbf{B}}^{i j}{ }_{\dot{X}}\right)+\mathbf{S}_{i} \mathbf{X}_{i} & =\bar{F}_{i, W} e^{-\mathrm{i} \omega t}+\mathbf{F}_{i, C}+\mathbf{F}_{i, R}, i=1, \ldots, N, \\
\mathbf{D}_{S i}^{R} \mathbf{X}_{i}-\mathbf{D}_{H(i+1)}^{R} \mathbf{X}_{i+1} & =0, \quad i=1,2, \ldots, N-1, \\
\mathbf{D}_{S i}^{L} \mathbf{X}_{i}-\mathbf{D}_{H(i+1)}^{L} \mathbf{X}_{i+1} & =0, \quad i=1,2, \mathrm{ev} \ldots, N-1 .
\end{aligned}
$$

The governing equation of motions for the multiple hinged floating structures is linear differential-algebraic equations (DAEs) which can be solved in the frequency domain.

\section{Hydrodynamic Responses of the Multiple Hinged Floating Structures}

3.1. Floating System with Hinged Connection. Two semisubmersible platforms connected by hinge connecter are considered in this paper, shown in Figure 5. Module M1 and module M2 are identical, and their detail parameters are listed in Table 1. The blue dots labeled with $\mathrm{C} 1$ and $\mathrm{C} 2$ denote the spherical hinge and pseudospherical hinge connectors, respectively. The transverse distance between the two hinge connectors is $\delta_{H}=20 \mathrm{~m}$, and the vertical distance of the hinge connector away from the center of gravity is $\delta_{V}=5 \mathrm{~m}$. The gap between adjacent modules is $\delta=1 \mathrm{~m}$.

In order to simplify the calculation, a rectangular parallelepiped rubber cushion is arranged between adjacent up hulls of the two modules. The rubber cushion is centered layout along the transverse direction with width $B_{r}=10 \mathrm{~m}$. The total height of the rubber cushion is $H_{r}=2 \mathrm{~m}$, whose distribution heights of the two sides along the vertical direction are $\delta_{V 1}, \delta_{V 2}$, where $H_{r}=\delta_{V 1}+\delta_{V 2}$.

In order to investigate the effect of topological properties of rubber cushion, a stiffness distribution function is introduced, given as



FIGURE 5: Sketch of 2-modular floating structure configuration.

Table 1: Principal characteristics of a single module.

\begin{tabular}{lcc}
\hline & Items & Values \\
\hline \multirow{3}{*}{ Upper hull } & Length $(\mathrm{m})$ & 30 \\
& Breadth $(\mathrm{m})$ & 25 \\
& Depth $(\mathrm{m})$ & 3.2 \\
\hline \multirow{4}{*}{ Columns } & Horizontal section $\left(\mathrm{m}^{2}\right)$ & $5.5 \times 5.5$ \\
& Depth $(\mathrm{m})$ & 7.5 \\
& Longitudinal spacing $(\mathrm{m})$ & 21 \\
& Transverse spacing $(\mathrm{m})$ & 20 \\
\hline \multirow{4}{*}{ Lower hull } & Length $(\mathrm{m})$ & 26 \\
& Breadth $(\mathrm{m})$ & 5.5 \\
& Depth $(\mathrm{m})$ & 3.5 \\
\hline \multirow{3}{*}{ Others } & Displacement $(\mathrm{t})$ & 1827 \\
& $I_{x}\left(\mathrm{~kg} \cdot \mathrm{m}^{2}\right)$ & $1.484 \times 10^{8}$ \\
& $I_{y}\left(\mathrm{~kg} \cdot \mathrm{m}^{2}\right)$ & $1.976 \times 10^{8}$ \\
& $I_{z}\left(\mathrm{~kg} \cdot \mathrm{m}^{2}\right)$ & $2.125 \times 10^{8}$ \\
\hline
\end{tabular}

$$
k(Y, Z)=k_{0} \Pi(Y) \Phi(Z)=\frac{k_{0}}{\sqrt{1+\Xi_{Z}\left(Z-Z_{0} / H_{r} / 2\right)^{2}} \sqrt{1+\Xi_{Y}\left(Y /\left(B_{r} / 2\right)\right)^{2}}},
$$

where $k_{0}$ is the stiffness coefficient at the center of the rubber cushion. The symbols $\Xi_{Y}, \Xi_{Z}$ indicate the distribution coefficients, which can determine the gradients of the stiffness along transverse and vertical directions, respectively.
3.2. Wave Spectrum for Irregular Sea. In this study, we use the standard JONSWAP spectrum $S(\omega)$ with choosing frequency spreading parameter $\gamma=3.3$ to simulate irregular waves:

$$
S(\omega)=\beta_{J} H_{s}^{2} T_{p}^{-4}\left(\frac{\omega}{2 \pi}\right)^{-5} \exp \left[-1.25\left(\frac{T_{p} \omega}{2 \pi}\right)^{-4}\right] \gamma^{\exp \left[-\left(T_{p} \omega / 2 \pi-1\right)^{2} / 2 \sigma^{2}\right]}
$$

where 


$$
\begin{aligned}
\beta_{J} & =\frac{0.06238}{0.230+0.0336 \gamma-0.185(1.9+\gamma)^{-1}[1.094-0.01915 \ln \gamma]}, \\
T_{p} & =\frac{T_{s}}{1-0.132(\gamma+0.2)^{-0.559},} \\
\sigma & = \begin{cases}0.07, & \omega \leq \omega_{p}, \\
0.09, & \omega>\omega_{p},\end{cases}
\end{aligned}
$$

where $T_{p}$ is the peak period. $H_{s}$ and $T_{s}$ are the significant wave height and significant wave period, respectively.

The 1-in-1000 maxima of the response are denoted as the extreme response amplitude $R_{e}$ for the short-term of any quantity, which is defined as [21]

$$
\begin{aligned}
R_{e} & =1.86 R_{s}=3.72 \sqrt{m_{0}}, \\
m_{0} & =\int_{0}^{\infty} H^{2}(\omega) S(\omega) d \omega
\end{aligned}
$$

where $R_{s}$ indicates the significant response amplitude, $m_{0}$ denotes the standard deviation, and $H(\omega)$ denotes the transfer function of the wave-response.

3.3. Numerical Validations. The numerical validations will be carried out first to show the feasibility of the method used in this study. In order to carry out the validations, the numerical results obtained by using the network method [22] which has been verified by experimental data are used to do the comparisons with those obtained in this study. In [22], a flexible connector is used to simulate the hinge connector between adjacent modules. Relative large stiffness $k_{x}=k_{y}=k_{z}=10^{10} \mathrm{~N} / \mathrm{m}$ for the flexible connector in [22] is selected to model the rigid hinge connector. The comparison of response amplitude operators (RAOs) in heave direction for the hinge platform without rubber cushion is shown in Figure 6. From Figure 6, we can see that the two results match well except for some frequency points with some minor discrepancies.

\subsection{Hydrodynamic Responses of Two-Module Hinged Floating} Structures in Regular Sea. Firstly, the effects of rubber cushion on the hydrodynamic responses of the multiple hinged floating structures are analyzed in regular waves. The stiffness distribution and the layout of the rubber cushion are illustrated by numerical simulation.

The response amplitude versus rubber stiffness is illustrated (shown in Figure 7) for constant stiffness distribution $\Xi_{Y}=0, \Xi_{Z}=0$ with wave parameters setting in wave frequency $\omega=0.9 \mathrm{rad} / \mathrm{s}$ and wave angle $\phi=45^{\circ}$.

From Figure 7, it can be seen that the roll, sway, and yaw motions are not affected by the rubber cushion because of the displacement constraints due to rigid hinged connectors, while the heave, surge, and pitch motions have rapidly changed due to the rubber cushion in the internal of $1 E 6<k_{0}<1 E 9 \mathrm{~N} / \mathrm{m}^{3}$. As the stiffness coefficient $k_{0}$ of the rubber cushion increases, the variation trends for the surge

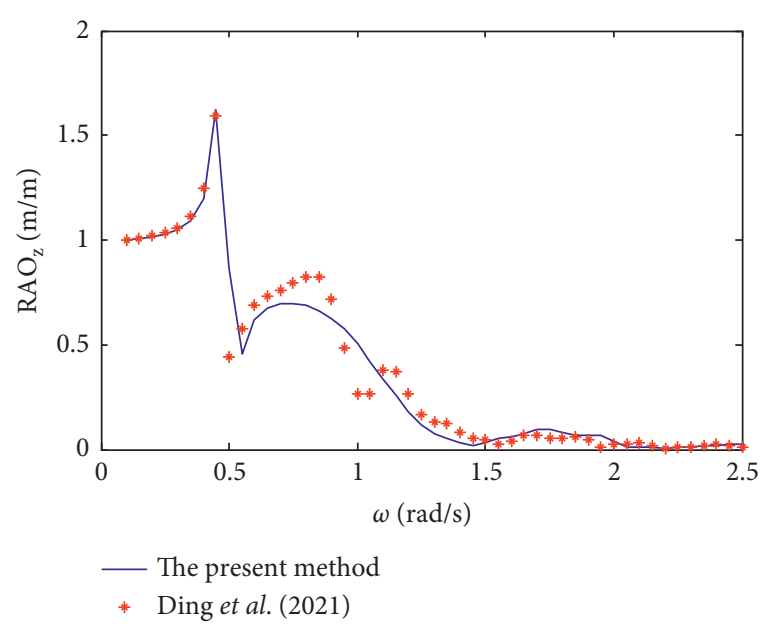

FIGURE 6: The motion response amplitude operators of heave motions for the results obtained by using the present method and Ding et al. with incident wave angle $\phi=0^{\circ}$.

and pitch motions are the same where the responses for the two adjacent modules reach almost similar values with the response of the first module decreasing and the response of the second module increasing. As to the heave motions, the responses of the two modules all increase firstly and subsequently reach a relatively stable value with the stiffness coefficient of the rubber cushion increasing. Two hinged floating modules behave similarly to the wings of a butterfly; the pitch motions of the modules, i.e., the flaps of wings, will lead to the surge and heave motions of the CG points of the modules in the viewpoint of the hinge connector. As the stiffness coefficient of the rubber cushion increases, the pitch degree of freedom will be reconstrained for the hinged floating structures. Thus, the decrease of the relative pitch motion due to the rubber cushion will decrease the relative surge motion. And the wave forces imposed on the pitch DOF of the floating modules will transfer to heave DOF which will increase the amplitude of the heave motions. We can conclude that the rubber cushion affects the pitch motions directly, while the relative heave and surge motions are indirectly affected by the variations of the relative pitch motion.

The $x, y$, and $z$ components of the magnitude of the force acting on the spherical hinge connector (C1) and pseudospherical hinge connector (C2) are plotted in Figure 8 as a function of stiffness coefficient $\left(k_{0}\right)$. From Figure 8 , we can see that rubber cushion only affects the longitudinal forces of the hinge connectors, and the variation trends of different connectors are the same. In the interval of $k_{0}<1 E 6 \mathrm{~N} / \mathrm{m}^{3}$ for the small stiffness of the rubber cushion, the longitudinal forces $F_{x}$ are similar to the forces without a rubber cushion. With the increases in the stiffness coefficient of rubber, the longitudinal forces of the hinge connectors rapidly change in the region of $1 E 6<k_{0}<1 E 9 \mathrm{~N} / \mathrm{m}^{3}$ and then reach relatively stable values.

After having carefully analyzed the above results and exhaustively examined other wave parameter settings (not included here), we can conclude that the rubber cushion installed between hinged floating modules can reduce the 


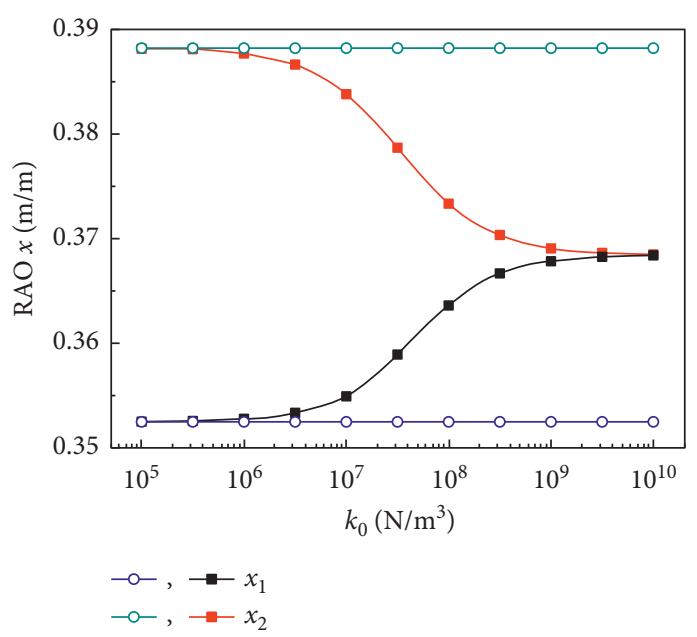

(a)

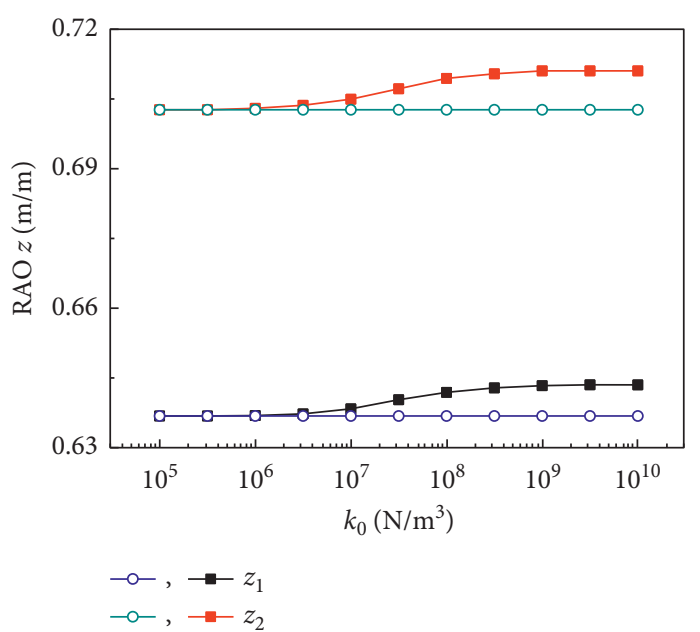

(c)

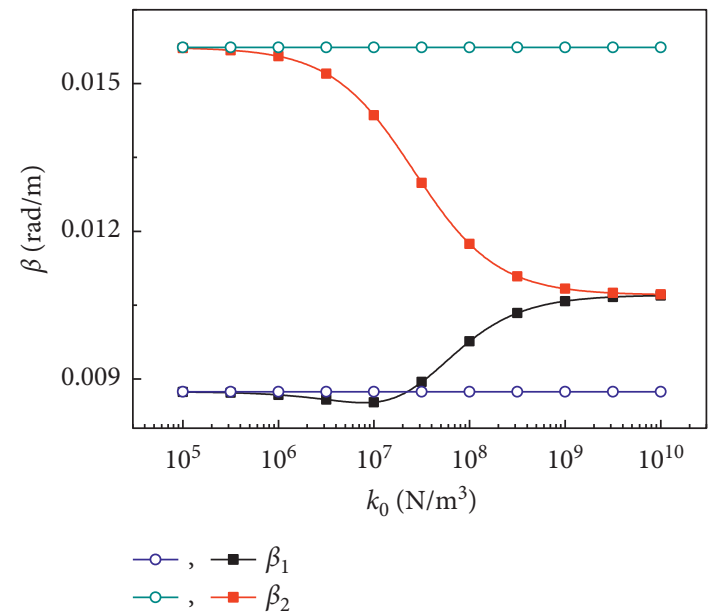

(e)

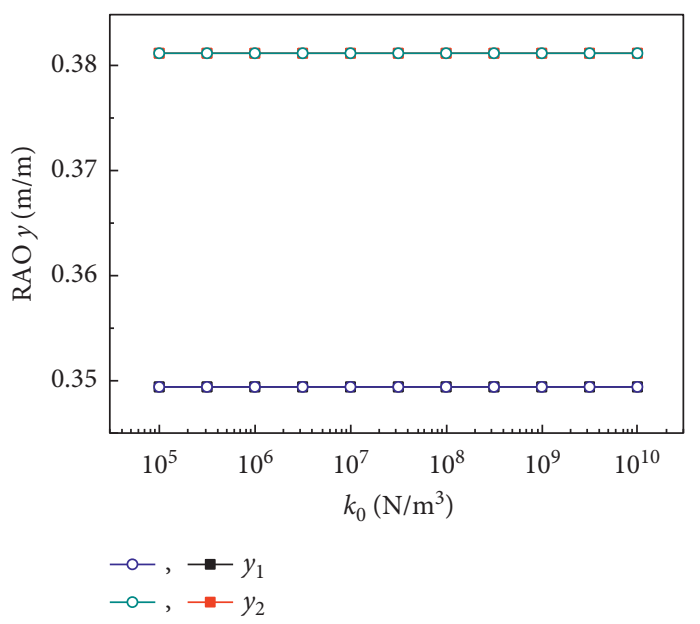

(b)

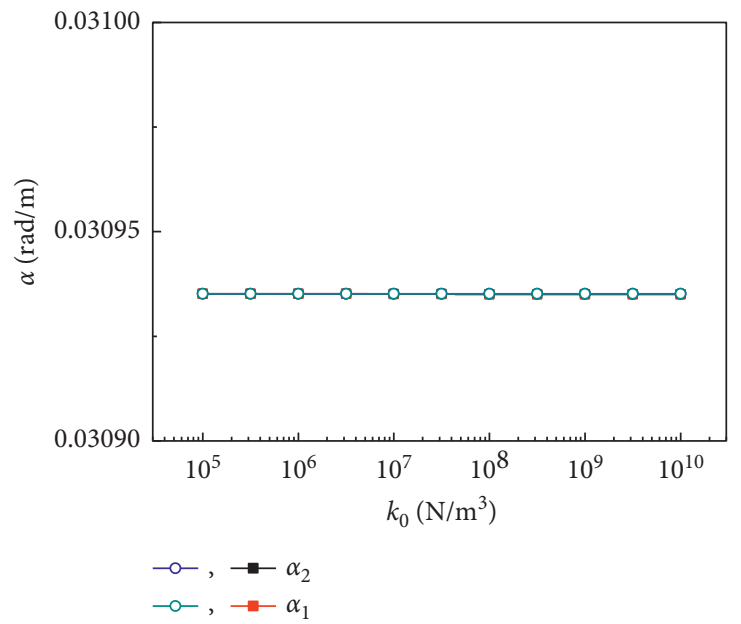

(d)

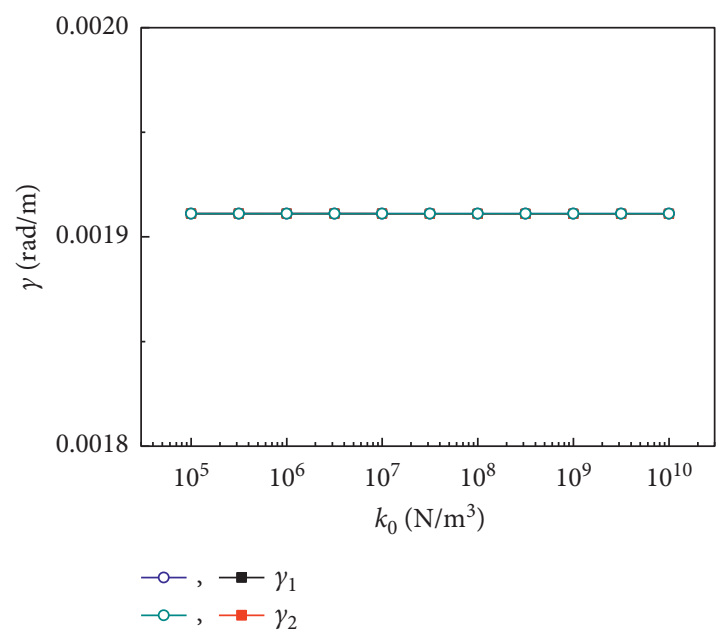

(f)

FIGURE 7: The response amplitude for all degrees of freedom versus stiffness of the rubber cushion for wave frequency $\omega=0.9 \mathrm{rad} / \mathrm{s}$, incident wave angle $\phi=45^{\circ}$, diamond line for results with rubber cushion, and circular line for results without rubber cushion.

relative pitch motion, but in the cost of increasing longitudinal forces of the hinge connectors. How to design the stiffness parameters of the rubber cushion to achieve a relatively optimum balance between the decrease of the relative pitch motion and the increase of the connector force is important. In the following, the influence performance of 


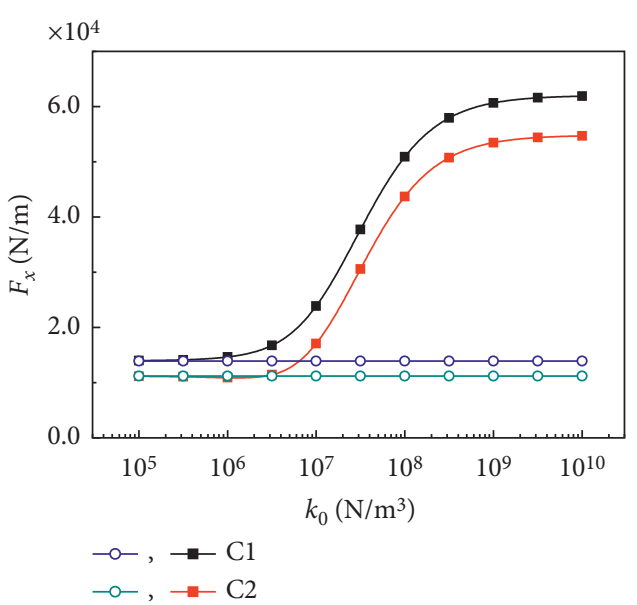

(a)



(b)

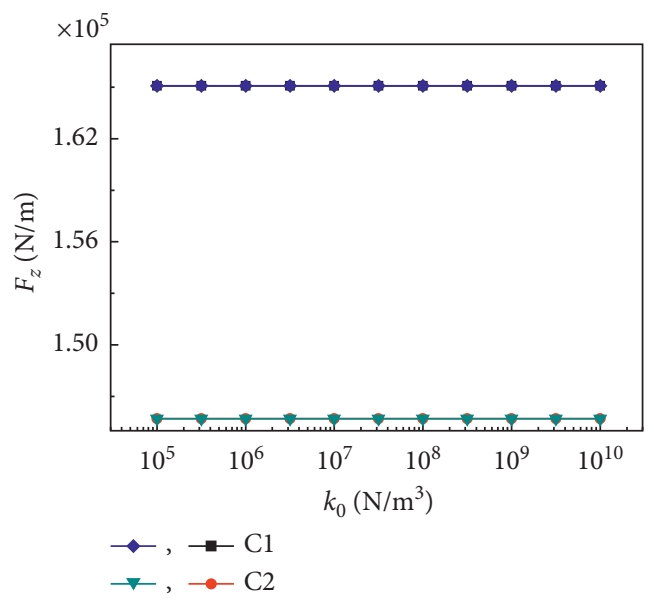

(c)

Figure 8: The force amplitude for the hinge connector versus stiffness of the rubber cushion (for wave frequency $\omega=0.9 \mathrm{rad} / \mathrm{s}$, wave angle $\phi=45^{\circ}$, diamond line for results with rubber cushion, and circular line for results without rubber cushion).

the stiffness distribution and the layout of the rubber cushion will be carried out.

Firstly, the stiffness distribution of the rubber cushion is analyzed via three different distribution forms shown in Figure 9: (1) uniform stiffness along two directions (red line), (2) stiffness variation only along the transverse direction (black line), and (3) stiffness variation only along the vertical direction (black line). It is possible to design the variable stiffness by hollowing the internal topological structure of the rubber cushion.

Figures 10 (a) and 10(b) show the variation of response amplitude of the relative pitch motion and longitudinal force acting on the connector $\mathrm{C} 1$, respectively, as a function of the stiffness of the rubber cushion for different stiffness distribution forms. It is observed that both $\Delta \beta$ and $F_{x}$ vary significantly in the range of our interest of stiffness $k_{0}=10^{5}-10^{10}$. With the increase of the stiffness $\left(k_{0}\right), \Delta \beta$ decreases continuously and becomes constant for $k_{0}>10^{9}$. The longitudinal force follows the reverse pattern of $\Delta \beta$ (Figure 10(b)). Stiffness distribution has a negligible effect on both $\Delta \beta$ and $F_{x}$ for $10^{6}<k_{0}<10^{9}$.

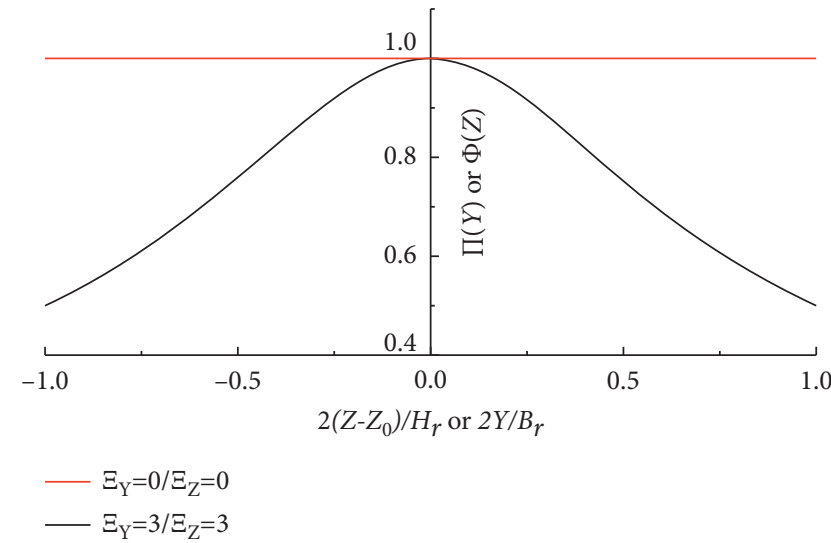

FIGURE 9: Stiffness distribution functions for different distribution coefficients.

However, constant values are found for $k_{0}<10^{6}$ and $k_{0}>10^{9}$. The uniform stiffness distribution has a weak superiority where the relatively small stiffness can obtain the same relative variation in comparison with different 


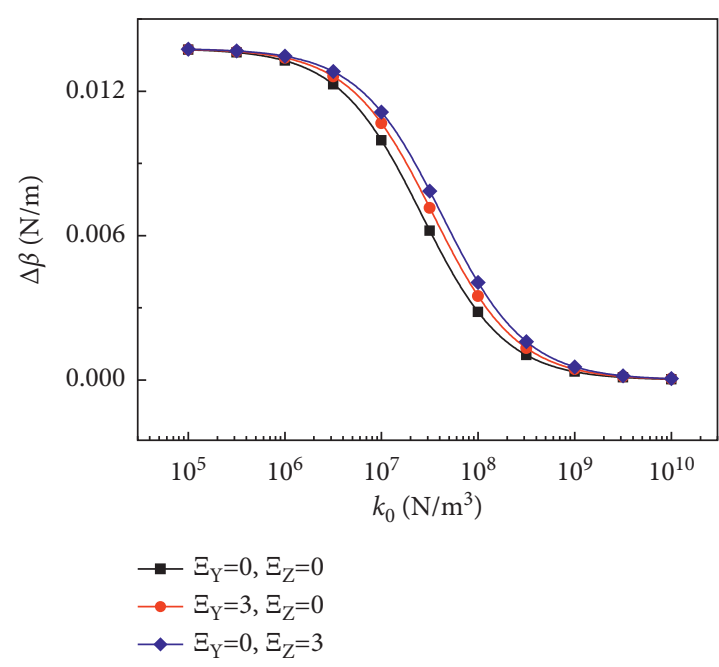

(a)



(b)

Figure 10: The response amplitude for the relative pitch angle between adjacent modules and horizontal force for hinge connector C1 versus stiffness of the rubber cushion (for wave frequency $\omega=0.9 \mathrm{rad} / \mathrm{s}$ and wave angle $\phi=45^{\circ}$ ).

distribution forms. For simplicity, in the following context, the uniform stiffness distribution is used for numerical simulation unless special specified.

In this paper, the rubber cushion is assumed symmetrically installed along the longitudinal midline. Considering the limited installation area of the upper hull and the total height of the rubber cushion, the installing height of the rubber cushion should be optimized to obtain better performance for the rubber cushion. For this purpose, a decentration ratio index $\Lambda$ relative to the hinge point along the vertical direction is introduced for simulation.

$$
\Lambda=\frac{\delta_{v 1}-\delta_{v 2}}{\delta_{v 1}+\delta_{v 2}}
$$

Examining the definition of the decentration ratio index in equation (36), the index $\Lambda<0$ denotes the rubber cushion installed partially to the upside of the hinge point, while the index $\Lambda>0$ denotes the rubber cushion installed partially to the downside of the hinge point. Figure 11 illustrates the response amplitudes for the relative pitch angle between adjacent modules and horizontal force for hinge connector $\mathrm{C} 1$ versus decentration ratio index with parameter setting in wave frequency $\omega=0.9 \mathrm{rad} / \mathrm{s}$ and wave angle $\phi=45^{\circ}$.

It can be seen from Figure 11 that the decentration ratio index has the almost opposite effect on the relative pitch angle between adjacent modules and horizontal force for the hinge connector. When the rubber cushion installs symmetrically based on the center of the hinge point along the vertical direction, the constraint of the relative pitch motion is the weakest, while the effect of rubber cushion on the longitudinal force of the hinge connector reaches the minimum. As the decentration ratio increases, the relative pitch angle of the modules decreases while the surge force of the connector increases. The variation gradient of the surge force is obviously larger than that of the relative pitch angle. Based on the analysis above, the rubber cushion installed

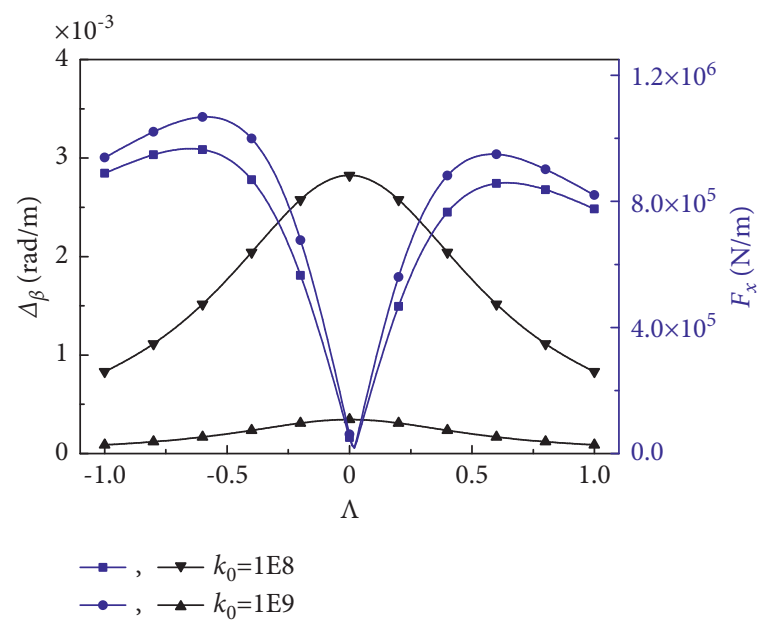

FIgURE 11: The response amplitude for the relative pitch angle between adjacent modules and longitudinal force for hinge connector $\mathrm{C} 1$ versus decentration ratio index (for wave frequency $\omega=$ $0.9 \mathrm{rad} / \mathrm{s}$ and wave angle $\phi=45^{\circ}$ ).

symmetrically based on the center of the hinge point is also a superior option where the surge force of the hinge connector is amplified weakly; meanwhile, the relative pitch motion of the modules can be constrained effectively to guarantee smoothness among adjacent modules.

In the following, the effects of the symmetrical rubber cushion appear on the resonance peaks of the dynamic responses for the floating structures. Figure 12 illustrates the amplitude-frequency response curves for the pitch DOF of the first module and the longitudinal connector load of the $\mathrm{C} 1$ connector. From Figure 12, we can see that the resonance peaks of response for pitch DOF decrease obviously in the low frequency range when stiffness coefficient of rubber cushion $k_{0} \geq 1 E 7 \mathrm{~N} / \mathrm{m}^{3}$. Out of the low frequency range, the 




(a)

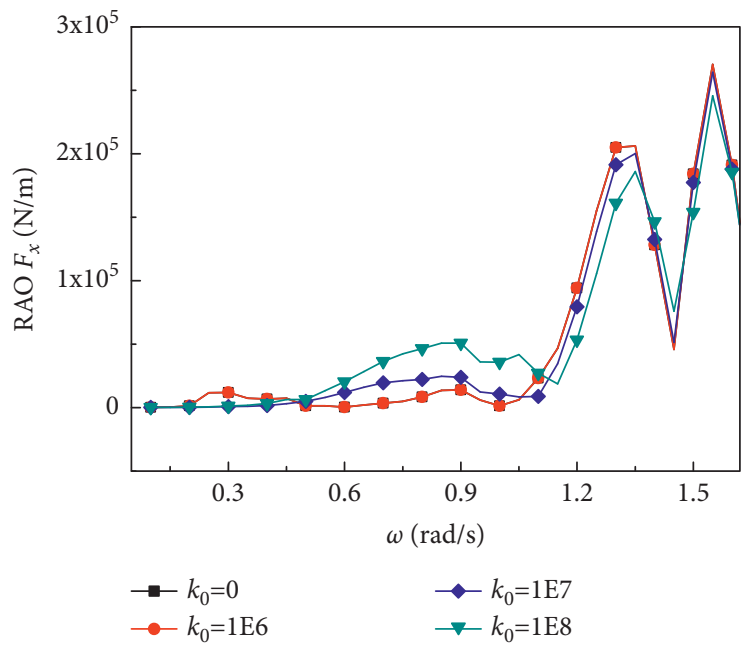

(b)

Figure 12: The transfer function for pitch motion of module 1 and connector force of hinge connector $\mathrm{C} 1$ for different stiffness coefficients of rubber cushion (for wave angle $\phi=45^{\circ}$ ).

effect of different stiffness coefficients on the resonance peaks is insignificant although the resonance peaks can be decreased slightly with the increasing stiffness coefficient of the rubber cushion. The resonance peak of the connector load locates in the high wave frequency range and the effect of the stiffness coefficient in this range is insignificant. The effect of the stiffness coefficient on the connector load is obvious in the low frequency range which can be ignored because the connector load in this range is much less than the design loads.

3.5. Extreme Responses for Hinged Floating Structures in Irregular Sea. In this study, the target sea area selected for this platform is near a typical island in the South China Sea. The design sea state $\left(H_{s}=3 \mathrm{~m}, T_{p}=8 \mathrm{~s}\right)$ in this manuscript is based on the measured analysis data. Figure 13 illustrates the extreme responses of six degrees of freedom for module 1 in the parameter space $\left(\phi, k_{0}\right)$ for significant wave height $H_{s}=3 \mathrm{~m}$ and peak wave period $T_{p}=8 s$. As shown in Figure 13, the rubber cushion has only an effect on the degrees of freedom in surge $x_{1}$, heave $z_{1}$, and pitch $\beta_{1}$ and the variations for surge and heave motions are very weak and ignorable when the stiffness coefficient for the rubber cushion increases. For the different incident wave angles, the pitch motion has the same variation trend that pitch angle decreases with increases in stiffness coefficient of rubber cushion. When the stiffness of the rubber cushion is larger than $1 E 8 \mathrm{~N} / \mathrm{m}^{3}$, the pitch angle will reach a constant value.

Figure 14 illustrates the extreme loads of the connector $\mathrm{C} 1$ in the parameter space $\left(\phi, k_{0}\right)$ for significant wave height $H_{s}=3 \mathrm{~m}$ and peak wave period $T_{p}=8 \mathrm{~s}$. From Figure 14, we can see that the longitudinal load of the connector is only affected by the rubber cushion. Similarly, with the variation of pitch motion, the variation trends for connector load are the same for different incident wave angles.
Figure 15 illustrates the extreme response of the relative pitch angle in the parameter space $\left(\phi, k_{0}\right)$ when the significant wave height $H_{s}=3 \mathrm{~m}$ and peak wave period $T_{p}=8 \mathrm{~s}$. For arbitrary incident wave angle, the extreme response of the relative pitch motion decreases rapidly as the stiffness coefficient of the rubber cushion increases. In comparison with the extreme response of the pitch motion shown in Figure 13(e), we can see that the slope of the surface along the variation direction of stiffness coefficient for the relative pitch motion is much larger than that for the pitch motion, which indicates that the relative pitch motion is constrained while the pitch motion for the two modules as an integral will increase with installing rubber cushion. Similarly, in comparison with the extreme longitudinal load of the connector C1 shown in Figure 14(a), we can see that the slope of the surface along the variation direction of stiffness coefficient for the relative pitch motion is much larger than that for the extreme longitudinal load, which can qualitatively indicate that the relative pitch motion can be ideally constrained to improve the smoothness between adjacent modules by installing rubber cushion with slightly increasing design load for the connector.

Figure 16 shows the extreme response of the relative pitch motion and the longitudinal force versus stiffness coefficient of the rubber cushion in variable wave conditions. It can be seen from Figure 16 that the extreme response of relative pitch motion decreases rapidly while the extreme load of longitudinal increases in the range of $1 E 6<k_{0}<1 E 8 \mathrm{~N} / \mathrm{m}^{3}$. For different wave conditions, the variation trends for the extreme response of the relative pitch angle and the longitudinal load are similar while the effectiveness of the constraint on the relative pitch motion is much better in larger significant wave height conditions.

In order to quantify the circumstance of constraining for relative pitch motion and amplifying for the longitudinal load, a variation ratio $\eta$ is introduced: 


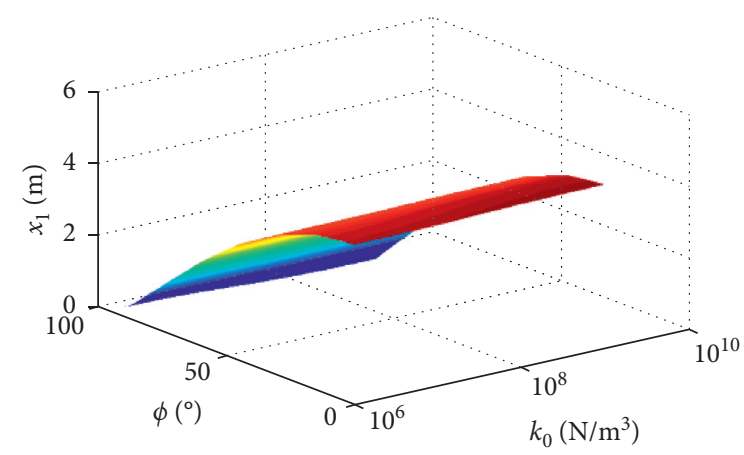

(a)

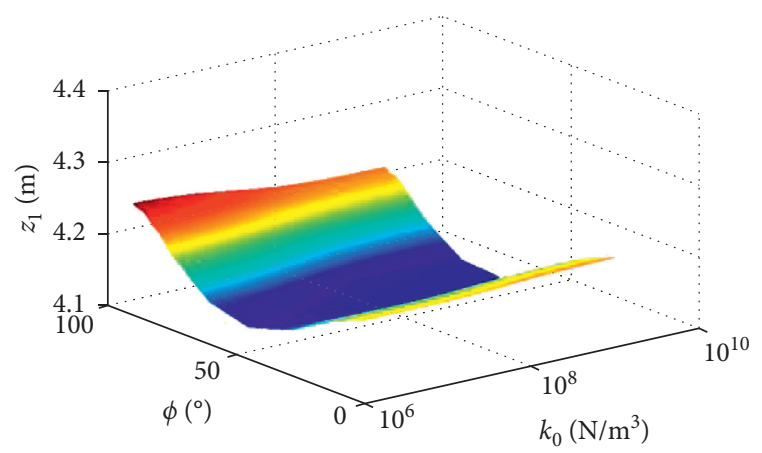

(c)



(e)

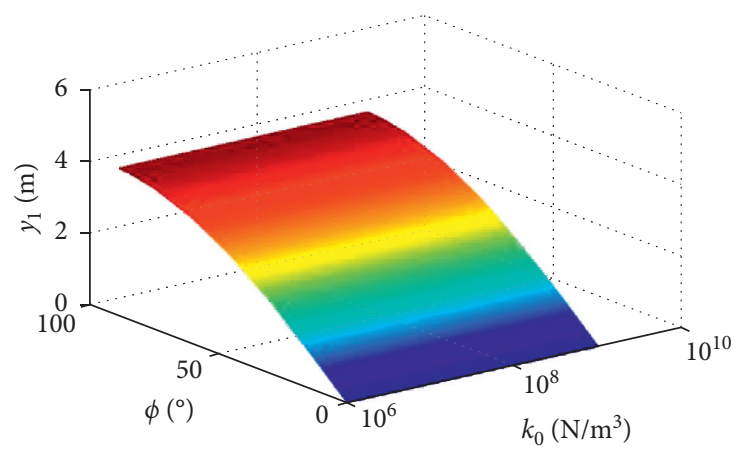

(b)



(d)

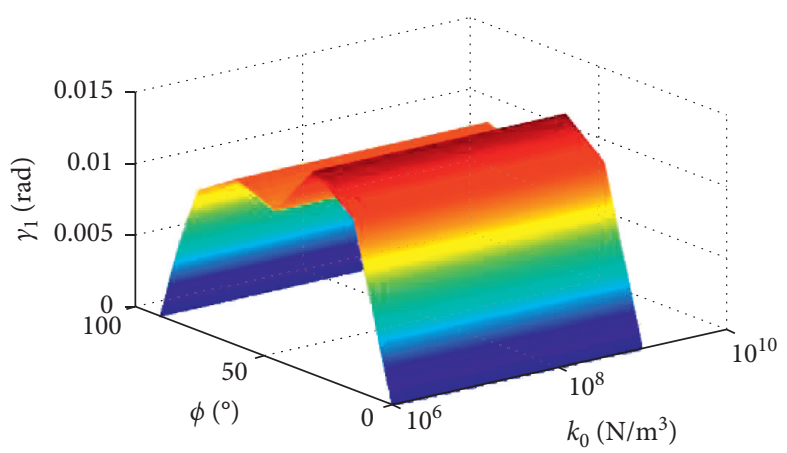

(f)

FiguRE 13: The extreme responses for all degrees of freedom of module 1 in parameter space $\left(\phi, k_{0}\right)$ for significant wave height $H_{s}=3 m$ and peak wave period $T_{p}=8 s$.

$$
\eta_{A}=\left|\frac{A-A_{0}}{A_{0}}\right|,
$$

where $A, A_{0}$ indicate the values for the argument with or without rubber cushion, respectively.

Figure 17 shows the variation ratios of the relative pitch angle and the longitudinal load versus the stiffness coefficient of the rubber cushion. From Figure 17, we can obviously see that the suppression ratio for the relative pitch motion is larger than the amplification ratio for the longitudinal load of the connector. In theory, the relative pitch motion can be constrained completely via only the increase of about $20 \%$ of longitudinal load by means of installing a rubber cushion in the extreme case. For a certain engineering design for rubber cushion, we should choose an appropriate stiffness for the rubber cushion which reaches an optimal balance for the effective constraint of the relative pitch angle and desirable increase of connector load. For example, with stiffness coefficient setting in $k_{0}=1 \mathrm{E} 7 \mathrm{~N} / \mathrm{m}^{3}$, the relative pitch motion can be constrained by $45 \%$ while the extreme load of the connector only increases about $5 \%$.

In order to estimate the feasibility for practical use, the strength of the rubber cushion is investigated. Considering the equivalent stiffness for the rubber cushion in equation (28), the simplified strain and stress of the rubber cushion between the $i$-th module and the $j$-th module can be formulated as

$$
\begin{gathered}
\varepsilon_{r}=\frac{\left[\left(x_{j}-x_{i}\right)+Z\left(\beta_{j}-\beta_{i}\right)-Y\left(\gamma_{j}-\gamma_{i}\right)\right]}{\delta}, \\
\sigma_{r}=k_{0}\left[\left(x_{j}-x_{i}\right)+Z\left(\beta_{j}-\beta_{i}\right)-Y\left(\gamma_{j}-\gamma_{i}\right)\right] .
\end{gathered}
$$




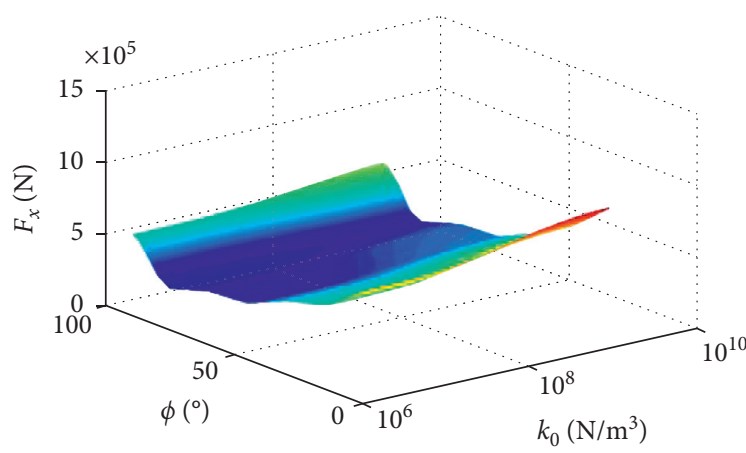

(a)

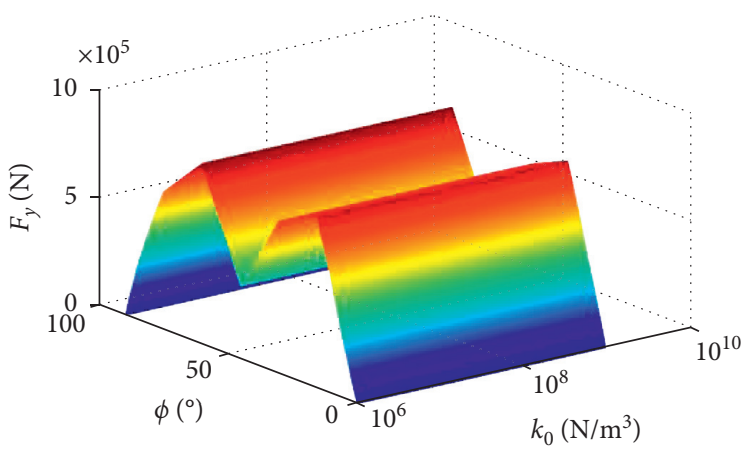

(b)

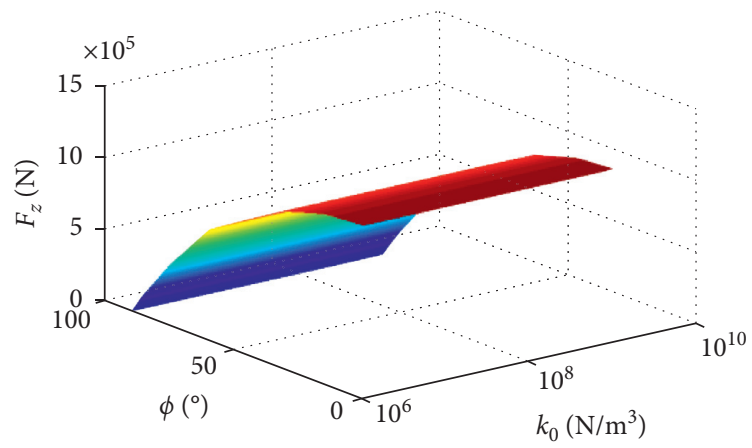

(c)

Figure 14: The extreme loads for connector $\mathrm{C} 1$ in parameter space $\left(\phi, k_{0}\right)$ for significant wave height $H_{s}=3 \mathrm{~m}$ and peak wave period $T_{p}=8 s$.

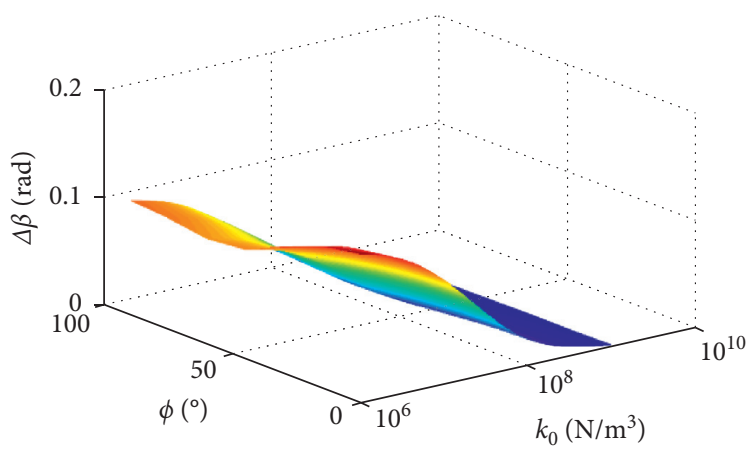

FIGURE 15: The extreme response of the relative pitch angle between adjacent modules in parameter space $1 E 8 \mathrm{~N} / \mathrm{m}^{3}$ for significant wave height $H_{s}=3 \mathrm{~m}$ and peak wave period $T_{p}=8 \mathrm{~s}$.

Figure 18 shows the strain and stress diagram for the rubber cushion with the stiffness coefficient of the rubber cushion $k_{0}=1 E 7 \mathrm{~N} / \mathrm{m}^{3}$ and wave angle $\phi=45^{\circ}$.

From Figure 18, we can see that the distribution forms of the strain and stress contour for the rubber cushion are symmetrical with respect to the horizontal plan $(Z=5 \mathrm{~m})$ where hinge connectors are installed. Considering the constraint conditions of the hinge connectors, the relative motions in surge and yaw directions are zeros, and thus, the strain and stress of the rubber cushion are only determined by the relative pitch motion. So, the strain and stress of the rubber cushion distribute linearly along the vertical direction. The maximum strain and stress of the rubber cushion are 0.07 and $0.7 \mathrm{MPa}$, respectively, which are much less than the allowable strain $[\varepsilon]=1.5$ and stress $[\sigma]=10 \mathrm{MPa}$ of the rubber material.

In fact, the stress distribution of the rubber cushion is determined by the stiffness distribution defined by equation (39). Thus, the stiffness distribution effect on the stress of the rubber cushion is analyzed hereinafter. Figure 19 illustrates the stress contour of the rubber cushion for three different distribution forms shown in Figure 9 for stiffness coefficient $k_{0}=1 E 7 \mathrm{~N} / \mathrm{m}^{3}$ and wave angle $\phi=45^{\circ}$.

From Figure 19, we can know that choosing different stiffness distribution forms has a remarkable influence on 


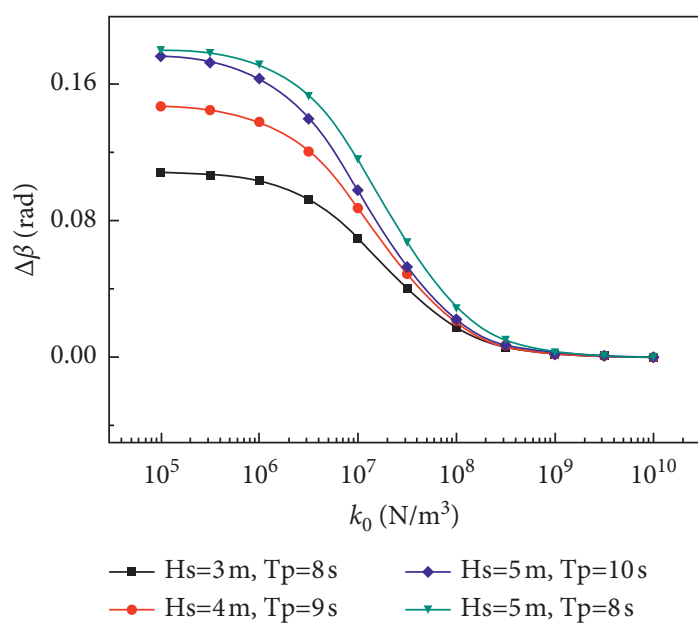

(a)

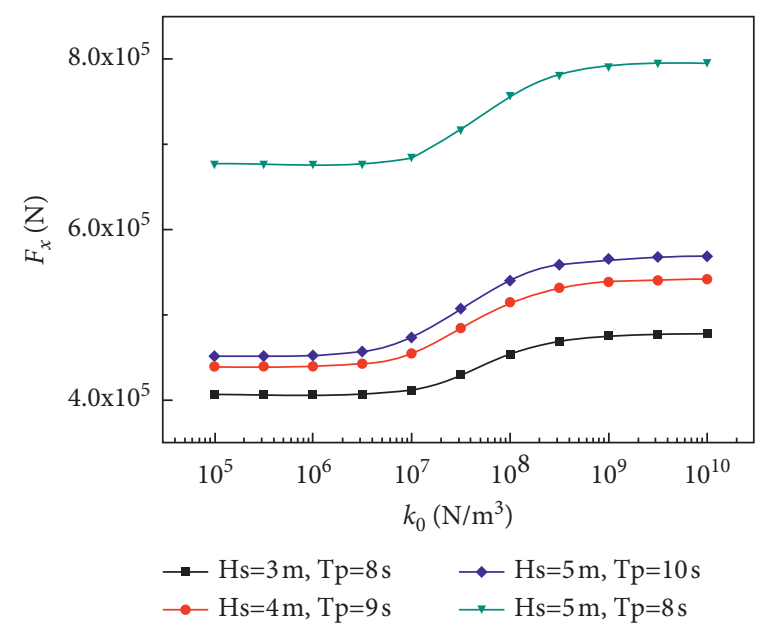

(b)

FIGURE 16: The extreme response for the relative pitch angle between adjacent modules and the longitudinal load of connector $\mathrm{C} 1$ for wave angle $\phi=45^{\circ}$.

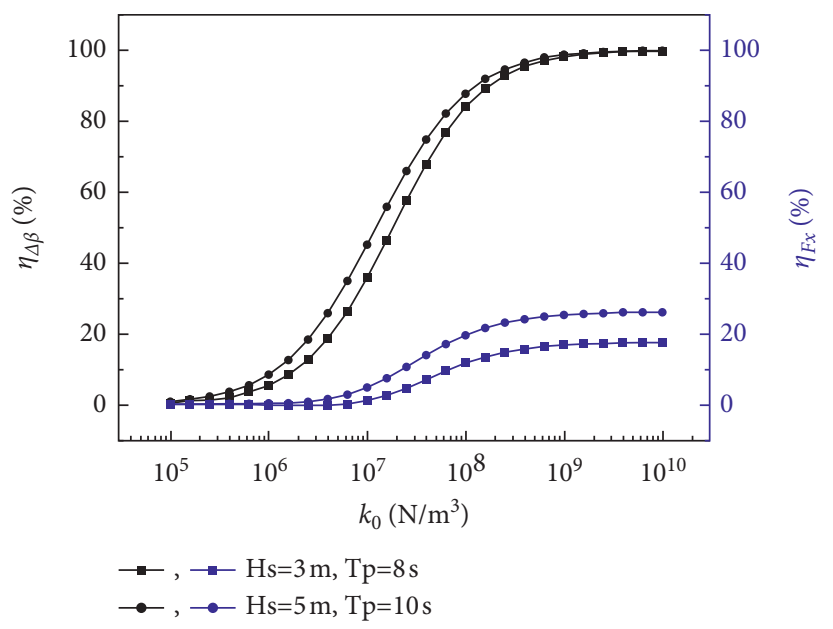

FIGURE 17: The rate of change for the relative pitch angle between adjacent modules (black lines) and the horizontal load of connector C1 (blue lines) versus stiffness coefficient of rubber cushion for wave angle $\phi=45^{\circ}$.

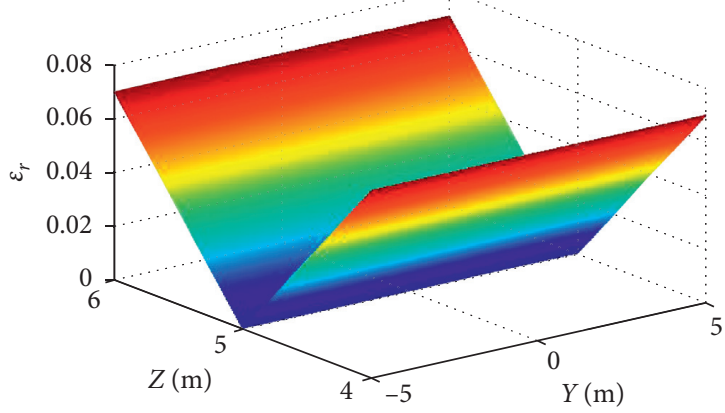

(a)

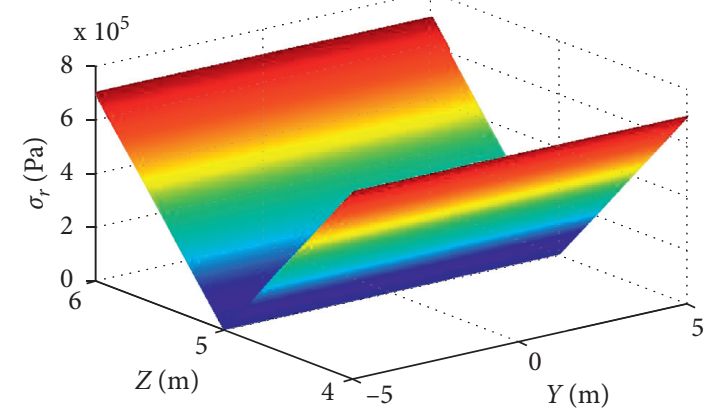

(b)

FIGURE 18: The 3D contour of strain and stress for the rubber cushion with the stiffness coefficient of the rubber cushion $k_{0}=1 E 7 \mathrm{~N} / \mathrm{m}^{3}$, wave angle $\phi=45^{\circ}$, significant wave height $H_{s}=3 \mathrm{~m}$, and peak wave period $T_{p}=8 \mathrm{~s}$. 


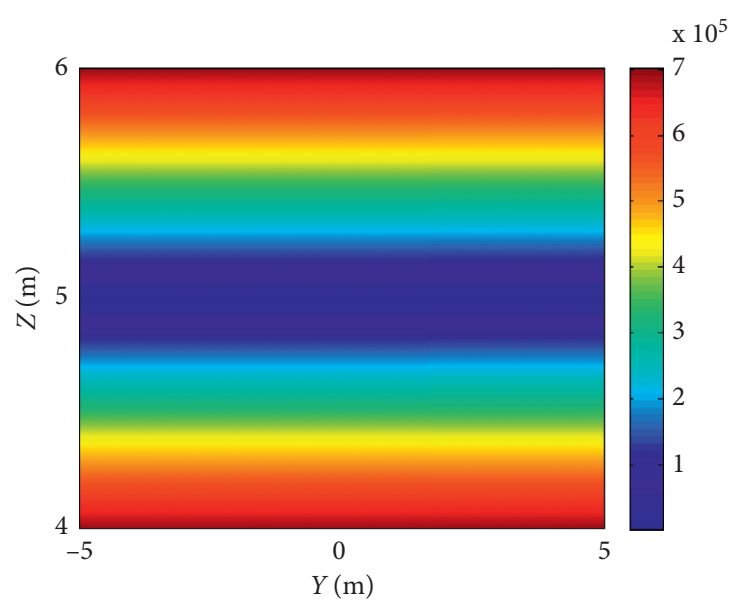

(a)

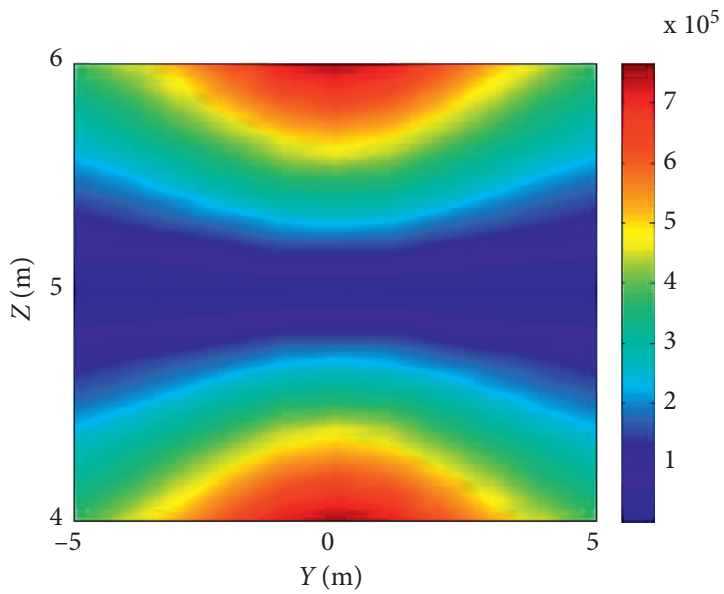

(b)

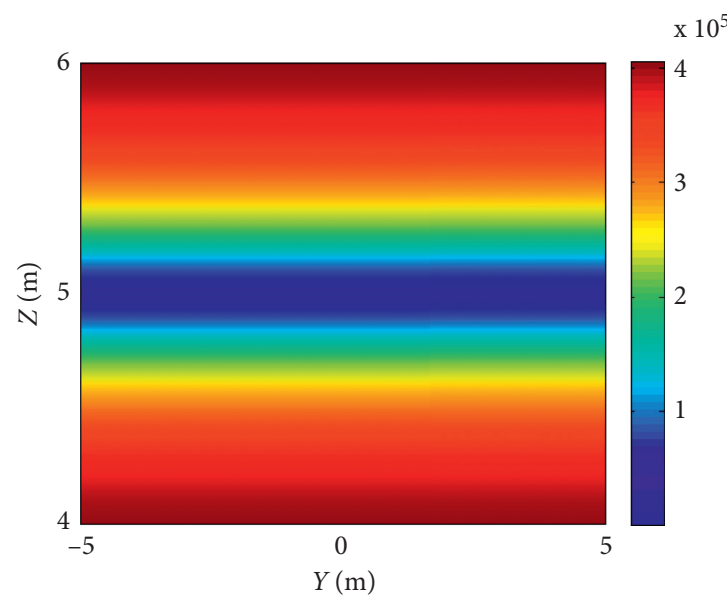

(c)

FIGURE 19: The stress contour of the rubber cushion for three different distribution forms ((a) $\Xi_{Y}=0, \Xi_{Z}=0$, (b) $\Xi_{Y}=3, \Xi_{Z}=0$, and (c) $\Xi_{Y}=0, \Xi_{Z}=3$ ) for stiffness coefficient $k_{0}=1 E 7 \mathrm{~N} / \mathrm{m}^{3}$ and wave angle $\phi=45^{\circ}$.

the stress of the rubber cushion. The maximum stresses for the three stiffness distribution forms are $0.7 \mathrm{MPa}, 0.76 \mathrm{MPa}$, and $0.4 \mathrm{MPa}$, respectively, which means that the stiffness variation along the transverse direction can remarkably improve the stress level of the rubber cushion. However, the conclusion obtained from Figure 10 is that the different stiffness distribution forms have a weak effect on the variations of the relative pitch motion and longitudinal force. When we reexamine the stress diagram shown in Figure 19, we can see that the red region, i.e., high-stress region, in Figure 19(c) is larger than that in Figures 19(a) and 19(b), which means that the effective area of the bearing load is larger for the rubber cushion with stiffness variation along the transverse direction. So, the total constraint loads for the hinged structure integrated from the whole rubber area change weakly for different stiffness distribution forms. In other words, the utilization lever for the rubber cushion can be remarkably improved via choosing appropriate stiffness distribution forms and the optimization design should be carried out for a specific connector.

\section{Conclusions}

This paper is concerned with the dynamic effects of the hinged floating structures when installing rubber cushions between adjacent modules. The dynamic model is formulated in which an equivalent stiffness matrix for the rubber cushions is derived by an integrating method based on the linear assumption in addition to considering the heterogeneity of rubber. A numerical example of a twomodule semisubmersible structure is illustrated. The effects of the rubber cushion on the hydrodynamic responses and the connector loads of the hinged floating structures are analyzed using the frequency domain approach in regular and irregular waves. The topological design and stiffness parameter selection of the rubber cushion is evaluated. The numerical results show that installing a rubber cushion can remarkably reduce the relative pitch motion to ensure the smoothness between adjacent modules via appropriately increasing the design longitudinal load of the hinge connector. The strength of the cushion satisfied the allowance stress of the rubber 
material. Nevertheless, we have to note that only numerical simulations are carried out in this manuscript, and the wave basin test will be conducted in our future work [23].

\section{Data Availability}

The data used to support the findings of this study are available from the corresponding author upon request.

\section{Conflicts of Interest}

The authors declare that they have no conflicts of interest.

\section{Acknowledgments}

This research work was supported by the National Natural Science Foundation of China, China (51809241 and 11702088) and the High-tech Ship Research Projects sponsored by MIIT and the Ministry of Science and Technology (Grant no. 2017YFB0202702).

\section{References}

[1] T. Utsunomiya, T. Moan, E. Watanabe, and C. M. Wang, Very Large Floating Structures: Applications, Analysis and Design, National University of Singapore, Singapore, 2004.

[2] H. Zhang, D. Xu, C. Lu, E. Qi, J. Hu, and Y. Wu, "Amplitude death of a multi-module floating airport," Nonlinear Dynamics, vol. 79, no. 4, pp. 2385-2394, 2015.

[3] H. C. Zhang, D. L. Xu, C. R. Liu, and Y. S. Wu, "Wave energy absorption of a wave farm with an array of buoys and flexible runway," Energy, vol. 109, pp. 211-223, 2016.

[4] S. Kim and K. H. Lee, "Hydrodynamic analysis of floating structures with baffled ARTs," Structural Engineering \& Mechanics, vol. 68, no. 1, pp. 1-15, 2018.

[5] M. S. Chen, M. Y. Zou, and L. Zhu, "Frequency-domain response analysis of adjacent multiple floaters with flexible connections," Journal of Ship Mechanics, vol. 22, no. 9, pp. 1164-1180, 2018.

[6] D. Jiang, K. H. Tan, C. M. Wang, and J. Dai, "Research and development in connector systems for very large floating structures," Ocean Engineering, vol. 232, Article ID 109150, 2021.

[7] C. M. Wang and Z. Y. Tay, "Very large floating structures: applications, research and development," Procedia Engineering, vol. 14, pp. 62-72, 2011.

[8] E. Watanabe, T. Utsunomiya, C. M. Wang, and L. T. T. Hang, "Benchmark hydroelastic responses of a circular VLFS under wave action," Engineering Structures, vol. 28, no. 3, pp. 423-430, 2006.

[9] H. C. Zhang, D. L. Xu, C. Lu, E. R. Qi, C. Tian, and Y. S. Wu, "Connection effect on amplitude death stability of multimodule floating airport," Ocean Engineering, vol. 129, no. 1, pp. 46-56, 2017.

[10] J. N. Newman, Wave Effects on Hinged Bodies Part III-hinge Loads vs. Number of Modules, Technical Report, 1998.

[11] J.-S. Yoon, S.-P. Cho, R. G. Jiwinangun, and P.-S. Lee, "Hydroelastic analysis of floating plates with multiple hinge connections in regular waves," Marine Structures, vol. 36, pp. $65-87,2014$.
[12] C.-H. Lee and J. N. Newman, "An assessment of hydroelasticity for very large hinged vessels," Journal of Fluids and Structures, vol. 14, no. 7, pp. 957-970, 2000.

[13] K.-H. Jeong, "Hydroelastic vibration analysis of liquid-contained rectangular tanks," Structural Engineering \& $M e$ chanics, vol. 40, no. 5, pp. 665-688, 2011.

[14] S. Ohmatsu, "Numerical calculation method for the hydroelastic response of a pontoon-type very large floating structure close to a breakwater," Journal of Marine Science and Technology, vol. 5, no. 4, pp. 147-160, 2000.

[15] S. Y. Xia, D. L. Xu, H. C. Zhang, E. R. Qi, J. J. Hu, and Y. S. Wu, "On retaining a multi-module floating structure in an amplitude death state," Ocean Engineering, vol. 121, pp. 134-142, 2016.

[16] E. R. Qi, C. Liu, J. S. Xia, Y. Lu, Z. W. Li, and Y. L. Yue, "Experimental study of functional simulation for flexible connectors of very large floating structures," Journal of Ship Mechanics, vol. 19, no. 10, pp. 1245-1254, 2015.

[17] S. X. Fu, T. Moan, X. J. Chen, and W. C. Cui, "Hydroelastic analysis of flexible floating interconnected structures," Ocean Engineering, vol. 34, no. 11-12, pp. 1516-1531, 2007.

[18] M. Riyansyah, C. M. Wang, and Y. S. Choo, "Connection design for two-floating beam system for minimum hydroelastic response," Marine Structures, vol. 23, no. 1, pp. 67-87, 2010.

[19] C. Zhao, X. Hao, R. Liang, and J. Lu, "Influence of hinged conditions on the hydroelastic response of compound floating structures," Ocean Engineering, vol. 101, no. 1, pp. 12-24, 2015.

[20] F. El-Hawary, The Ocean Engineering Handbook, CRC Press, Boca Raton, FL, USA, 2001.

[21] H. R. Riggs, R. C. Ertekin, and T. R. J. Mills, "Impact of connector stiffness on the response of a multi-module mobile offshore base," International Journal of Offshore and Polar Engineering, vol. 9, pp. 126-133, 1999.

[22] R. Ding, D.-l. Yan, H.-c. Zhang et al., "An application of network modeling method to scientific research and demonstration platform-Connector load analysis," Journal of Hydrodynamics, vol. 33, no. 1, pp. 33-42, 2021. 